\title{
Utilizing High-temperature Atomic-resolution STEM and EELS to Determine Reconstructed Surface Structure of Complex Oxide
}

\author{
Weizong Xu${ }^{1}$, Preston C. Bowes ${ }^{1}$, Everett D. Grimley ${ }^{1}$, Douglas L. Irving ${ }^{1}$, and James M. LeBeau ${ }^{1}$ \\ 1. Department of Materials Science and Engineering, North Carolina State University, Raleigh, NC \\ 27695, USA
}

Surface reconstructions are conventionally characterized by scanning tunneling microscopy (STM), electron diffraction, or X-ray photoelectron spectroscopy (XPS) [1]. These methods indirectly determine the surface structure and chemistry, which requires subsequent structural refinement and confirmation by density functional theory (DFT). Complex oxide surface reconstructions not only involve atomic structural rearrangement, but also changes in surface stoichiometry or charge state. As the underlying crystal structure becomes more complicated, the surface tends to restructure into a variety of structures, affected by numerous factors such as surface polarization, temperature, and partial pressure. In the case when the surface structure changes with temperature, above indirect methods become limited [2]. A direct real-space characterization using scanning transmission electron microscopy (STEM) is thus ideal. However, it is still challenging to reveal surface atomic structure at high temperatures, particularly when additional surface chemical structure characterization is needed while maintaining the atomic-resolution.

In this talk, we present an in-situ method to directly determine the structure and chemistry of reconstructed surfaces combining with atomic-resolution HAADF-STEM, ABF-STEM and EELS. Critically, we will emphasize that these experiments are all conducted in the microscope and temperatures up to $900{ }^{\circ} \mathrm{C}$. A FIBless "in-situ by ex-situ" approach is used, which allows mounting a large pristine crystal onto Protochips membrane with minimum contamination for high-temperature characterization [3]. With HAADF-STEM imaging, we observe the following features of the reconstructed $\mathrm{SrTiO}_{3}(110)$ surface at $700{ }^{\circ} \mathrm{C}$ : (i) a new double-layer surface reconstruction occurring at $900{ }^{\circ} \mathrm{C}$ that becomes stable when it is cooled below $800{ }^{\circ} \mathrm{C}$, (ii) a periodic structure repeating every 5 $\mathrm{SrTiO}_{3}$ unit cells along [100] at $800{ }^{\circ} \mathrm{C}$ and below, and (iii) protruding atoms on top of the surface (Figure 1a). Although HAADF clearly resolves atom columns, the corresponding chemical information can only be unambiguously identified by atomic-resolution EELS. As seen in Figure 1b, Ti is the dominant element on double layers, while $\mathrm{Sr}$ is detected as the protruding atom column. Considering the similar contrast of protruding $\mathrm{Sr}$ and $\mathrm{Ti}$ column that cannot be distinguished in HAADF, atomicresolution EELS is thus required to interpret such complex surface structure.

We will also emphasize the use of multivariate statistical analysis (MSA) to improve the Ti and Sr maps in Figure 1b, which are reconstructed from the significant components in EELS spectrum image (SI) through MSA using custom MATLAB codes. The corresponding significant components are determined by non-negativity constrained multivariate curve resolution (MCR) algorithm employing constrained alternating least squares (ALS) [4]. Optimal scaling is conducted to process the SI data dealing with Poisson noise for MSA. The result of MSA clearly reveals the major Sr and Ti elemental distribution (component \#2 and \#4) in the atomic-resolution SI data other than the other statistically significant components from the spectrum average (\#1) and background (\#3). Figure 1c,d gives the spectrum profile of $\mathrm{Ti} \mathrm{L}_{3,2}$ and $\mathrm{O}-\mathrm{K}$ edge at each surface layer as indicated. Comparing the core loss fine structure of $\mathrm{Ti}$ and $\mathrm{O}$ with reference spectra, $\mathrm{Ti}^{+3}$ is found to co-exist with $\mathrm{Ti}^{+4}$ in the double layer, indicating the reduction of the excess charge on the polar (110) surface to stabilize the double layer structure. Utilizing 
the advantage of noise reduction via MSA, the charge distribution of Ti in the SI can be further obtained through multiple linear least squares (MLLS) fitting as shown in Figure 2a. Interestingly, Ti in +4 charges is found neighboring to the protruding atom columns. This finding suggests that $\mathrm{TiO}_{5}[]$ (where [] represents a vacant $\mathrm{Ti}$ octahedron site) forms locally, as identified from the ABF-STEM imaging for oxygen atoms columns in Figure $2 \mathrm{~b}$ and the subsequent DFT calculations. With the above information, we have demonstrated the capability of combining atomic-resolution EELS, HAADF and ABF-STEM in the determination of the reconstructed surface structures at high temperature. These structural details serve as a direct input for further first principles calculations.

\section{References:}

[1] J Enterkin et al, Nature Materials 9 (2010), p. 245.

[2] W Xu et al, Applied Physics Letters 109 (2016) p. 201601.

[3] W Xu et al, Microscopy and Microanalysis 22 (2016) p. 774.

[4] P Kotula, M Keenan and J Michael, Microscopy and Microanalysis 9 (2003) p. 1.

[5] The authors thank the support from the National Science Foundation (NSF) (DMR-1350273, DMR1151568), the Air Force Office of Scientific Research (FA9550-14-1-0182) and AIF at NCSU.
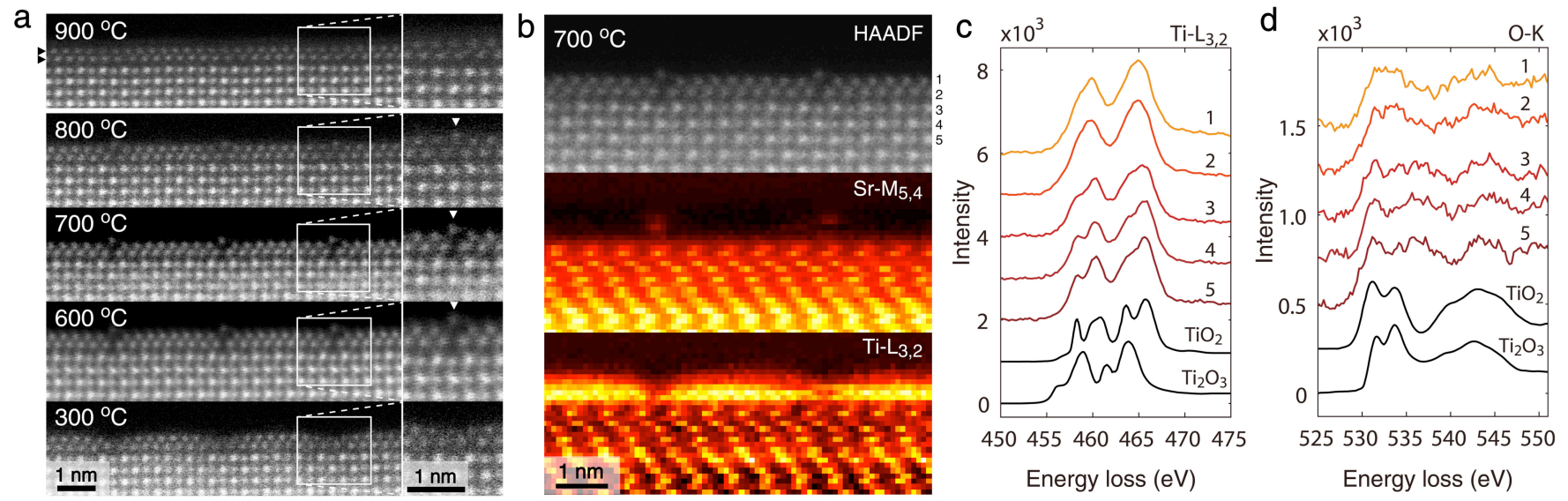

Figure 1. (a) HAADF-STEM images of reconstructed $\mathrm{SrTiO}_{3}(110)$ surface at indicated temperatures (b) HAADF and the MSA processed spectrum images of $\mathrm{Sr}$ and $\mathrm{Ti}$ at $700{ }^{\circ} \mathrm{C}$. Surface layer spectra of (c) $\mathrm{Ti}$ $\mathrm{L}_{3,2}$-edge and (d) O-K edge.
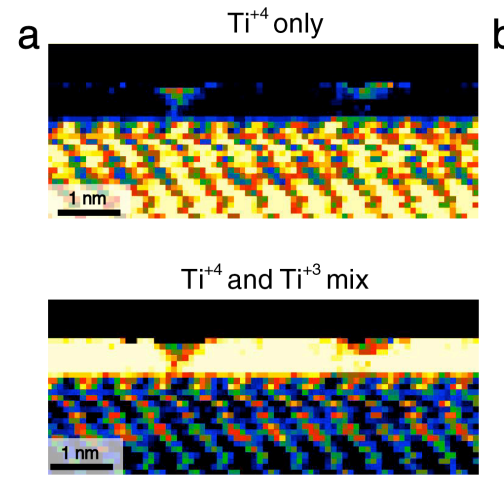

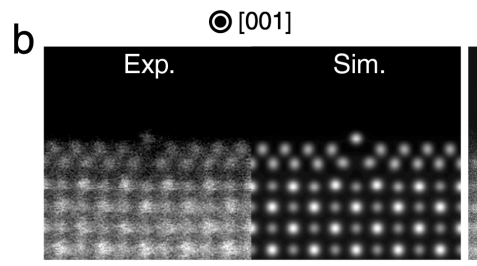

Exp.

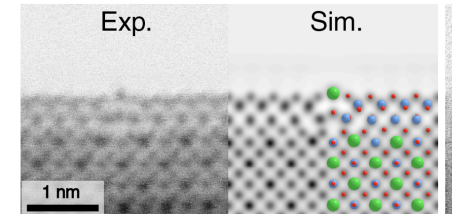

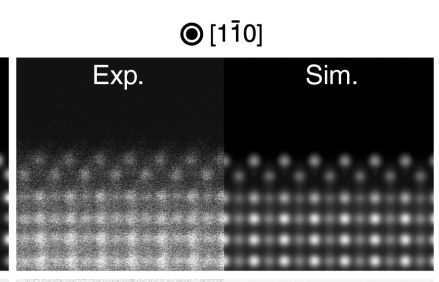

Sim.

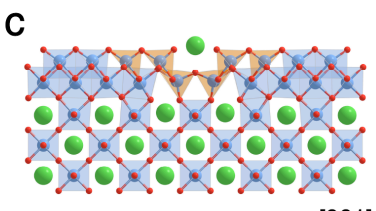

[001]

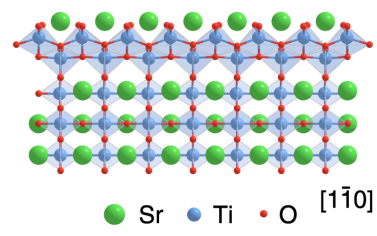

Figure 2. (a) Distribution maps of $\mathrm{Ti}^{+4}$ and $\mathrm{Ti}^{+4} / \mathrm{Ti}^{+3}$ mix. (b) Experiment observed and simulated HAADF and ABF-STEM images of the reconstructed double layers at $700{ }^{\circ} \mathrm{C}$. (c) Reconstructed surface models after DFT structural refinement. $\mathrm{TiO}_{5}[]$ structure is shown in orange color. 